TRANSACTIONS OF THE

AMERICAN MATHEMATICAL SOCIETY

Volume 352, Number 10, Pages 4501-4513

S $0002-9947(00) 02624-6$

Article electronically published on June 13, 2000

\title{
SYMPLECTIC 4-MANIFOLDS WITH HERMITIAN WEYL TENSOR
}

\author{
VESTISLAV APOSTOLOV AND JOHN ARMSTRONG
}

\begin{abstract}
It is proved that any compact almost Kähler, Einstein 4-manifold whose fundamental form is a root of the Weyl tensor is necessarily Kähler.
\end{abstract}

\section{INTRODUCTION}

An almost Kähler manifold is an almost Hermitian manifold for which the fundamental 2-form is closed, and therefore symplectic. If, additionally, the almost complex structure is integrable, we have a Kähler manifold.

The initial motivation for this paper is the conjecture due to Goldberg [16 that a compact almost Kähler, Einstein manifold is necessarily Kähler. This conjecture is still far from resolved, however, a number of results have been proved, mainly in dimension 4, when additional curvature conditions are added. Of course, these additional curvature conditions are necessarily conditions on the Weyl tensor of the manifold.

On an oriented Riemannian 4-manifold, the Weyl tensor decomposes under the action of $\mathrm{SO}(4)$ into two components - the so-called self-dual and anti-self-dual Weyl tensors, $W^{+}$and $W^{-}$. If, moreover, the manifold is almost Hermitian, then the self-dual Weyl tensor decomposes under the action of $\mathrm{U}(2)$ into three further pieces. So the self-dual Weyl tensor of an Einstein, almost-Hermitian 4-manifold has 3 interesting components. To see this, consider $W^{+}$as a trace-free self-adjoint map from the bundle $\Lambda^{+}$of self-dual 2-forms to itself. Since $\Lambda^{+}$decomposes under U(2) as $\mathbb{R} \oplus \llbracket \wedge^{0,2} \rrbracket$, we can write $W^{+}$as a matrix with respect to this block decomposition as follows:

$$
\left(\begin{array}{c|c}
\frac{\kappa}{6} & W_{2}^{+} \\
\hline\left(W_{2}^{+}\right)^{*} & W_{3}^{+}-\frac{\kappa}{12} \text { Id }
\end{array}\right),
$$

where the smooth function $\kappa$ is the so-called conformal scalar curvature, $W_{2}^{+}$corresponds to the part of $W^{+}$that interchanges the two factors of the U(2)-splitting of $\Lambda^{+}$, and $W_{3}^{+}$is a trace-free, self-adjoint endomorphism of the (real) rank 2 vector bundle $\llbracket \wedge^{0,2} \rrbracket$ underlying the anti-canonical bundle $\Lambda^{0,2}$.

Correspondingly, there are three special types of almost Hermitian, Einstein 4-manifolds each imposing one additional condition on the self-dual Weyl tensor:

1. $\kappa$ is constant (equivalently, the manifold has constant $*$-scalar curvature),

2. $W_{2}^{+}$vanishes (equivalently, the manifold is weakly $*$-Einstein),

Received by the editors June 21, 1999.

2000 Mathematics Subject Classification. Primary 53B20, 53C25.

The first author was supported in part by NSF grant INT-9903302.

(C)2000 American Mathematical Society 
3. $W_{3}^{+} \equiv 0$, (equivalently, the fundamental form is a root of the Weyl tensor). Manifolds with some of the above curvature properties constitute some of the most natural types of special almost Hermitian Einstein 4-manifolds. It is natural, therefore to attempt to prove the Goldberg conjecture with the addition of one or more of the above conditions.

In [3] it is shown that a compact, almost Kähler Einstein manifold satisfying condition (1) is necessarily Kähler. The equivalent result for condition (2) is shown in [4]. In fact a stronger result is proved: all non-Kähler, almost Kähler, Einstein 4-manifolds satisfying (2), even locally, are given by a construction due to Paul Tod. All these examples are in addition self-dual, i.e., $W^{-}$identically vanishes.

The purpose of this paper is to prove that compact almost Kähler, Einstein 4-manifolds satisfying (3) are necessarily Kähler.

Condition (3) arises naturally in the study of Hermitian geometry. On any Hermitian 4-manifold, the Weyl tensor automatically satisfies the condition $W_{3}^{+}=0$. For this reason we shall refer in this paper to manifolds satisfying this condition as having a "Hermitian Weyl tensor". Condition (3), however, does not imply the integrability of the almost Hermitian structure, but in certain circumstances knowledge of the Weyl tensor can be enough to show that an almost Hermitian structure is in fact Hermitian. For example, the Riemannian Goldberg-Sachs theorem (cf. [15, 20]) tells us that an Einstein 4-manifold admits a compatible Hermitian structure if and only if the self-dual Weyl tensor (viewed as a symmetric endomorphism of the self-dual 2-forms) is everywhere degenerate. In this case, the Hermitian structure produced will satisfy conditions (2) and (3).

The Riemannian Goldberg-Sachs theorem can be generalized from the situation of Einstein manifolds to manifolds whose Ricci tensor is $J$-invariant (i.e. it satisfies $\operatorname{Ric}(J \cdot, J \cdot)=\operatorname{Ric}(\cdot, \cdot))$; cf. [21], [1]. We shall show that our results generalize in a similar way. Specifically, we prove the following:

Theorem 1. Any compact almost Kähler 4-manifold $(M, g, J)$ with $J$-invariant Ricci tensor and Hermitian Weyl tensor is Kähler, provided that the Euler characteristic $e(M)$ and the signature $\sigma(M)$ of $M$ satisfy $5 e(M)+6 \sigma(M) \neq 0$.

For a compact (non-flat) Einstein 4-manifold the topological condition stated in Theorem 1 is verified as a consequence of the Hitchin-Thorpe inequality $2 e(M) \pm$ $3 \sigma(M) \geq 0$; we thus obtain:

Corollary 1. Any compact almost Kähler, Einstein 4-manifold with Hermitian Weyl tensor is Kähler.

Another application of Theorem 1 is given in Section 5 (Corollary 2) where a special class of self-dual, almost Kähler 4-manifolds is considered.

\section{Cartan-Kähler theory}

Suppose that one wishes to apply the Cartan-Kähler theory (as described in [17]) in order to prove that almost Kähler, Einstein metrics exist. One approach to the problem would be to take the standard symplectic form $\Omega$ on $\mathbb{R}^{4}$ and attempt to find metrics compatible with this form which are also Einstein. Here and henceforth, compatible means that the skew-symmetric endomorphism $J$, the dual of $\Omega$ with respect to the metric $g$, is an orthogonal almost complex structure, i.e., $(g, J, \Omega)$ is an almost Kähler structure. By Darboux's theorem, all almost Kähler, Einstein 
metrics occur in this way. Let us denote by $E$ the tangent space to the space of metrics compatible with $\Omega$. For any compatible almost Kähler structure $(g, J)$ the space $E($ at $(g, J))$ is identified with the real vector bundle of $J$-anti-invariant symmetric endomorphisms of the cotangent bundle $T^{*}$. We can then view the symbol of the Ricci operator as a map:

$$
S^{2} T^{*} \otimes E \stackrel{\sigma(\text { Ric })}{\longrightarrow} S^{2} T^{*} .
$$

One can easily check that this map is onto. This tells us that for any $\rho \in S^{2} T^{*}$ one can always find algebraic examples of 2 -jets of metrics compatible with $\Omega$ which satisfy $\operatorname{Ric}(g)=\rho$.

The next question is whether or not one can find algebraic examples of 3-jets satisfying this equation and its first derivative - i.e., whether one can find algebraic solutions to the first prolongation of the problem. The symbol of the first prolongation is the map

$$
S^{3} T^{*} \otimes E \stackrel{\sigma_{1}(\mathrm{Ric})}{\longrightarrow} T^{*} \otimes S^{2} T^{*} .
$$

One can easily check that this map is not onto. To see this consider that the differential Bianchi identity implies $\beta(\nabla \rho)=0$ for any solution of $\operatorname{Ric}(g)=\rho$, where $\beta: T^{*} \otimes S^{2} T^{*} \mapsto T^{*}$ is some equivariant map. The condition that $\beta(\nabla \rho)=0$ can be written entirely in terms of the 1-jets of our metric and $\rho$. Thus it imposes a condition that any 2-jet solution of the equation $\operatorname{Ric}(g)=\rho$ must satisfy if it is possible for it to be extended to a 3 -jet solution. The fact that the sequence

$$
S^{3} T^{*} \otimes E \stackrel{\sigma_{1}(\mathrm{Ric})}{\longrightarrow} T^{*} \otimes S^{2} T^{*} \stackrel{\beta}{\longrightarrow} T^{*} \longrightarrow 0
$$

is exact tells us that this is the only such condition. Note that exactly the same obstruction arises for the more general problem of finding metrics satisfying $\operatorname{Ric}(g)=\rho$ when one does not insist that the metric is compatible with the symplectic form. For the Einstein equation $\operatorname{Ric}(g)=\lambda g$, the obstruction simply tells us that $\lambda$ must be constant on the manifold.

One naturally wonders whether or not there are any higher order obstructions to extending $n$-jet solutions to the Einstein equations to $(n+1)$-jet solutions. The Cartan-Kähler Theorem gives a method of answering this question and it turns out, 14, that if one is looking for metrics $g$ not necessarily compatible with a symplectic form, then there are no further obstructions.

However, in the case that we are considering metrics compatible with $\Omega$, we find that the sequence

$$
S^{4} T^{*} \otimes E \stackrel{\sigma_{2}(\text { Ric })}{\longrightarrow} S^{2} T^{*} \otimes S^{2} T^{*} \stackrel{\sigma_{1}(\beta)}{\longrightarrow} T^{*} \otimes T^{*} \longrightarrow 0
$$

is not exact. By calculating the dimension of the image of $\sigma_{2}$ (Ric) one can see that there must be some equivariant map $\gamma: S^{2} T^{*} \otimes S^{2} T^{*} \longrightarrow \mathbb{R}$ such that

$$
S^{4} T^{*} \otimes E \stackrel{\sigma_{2}(\mathrm{Ric})}{\longrightarrow} S^{2} T^{*} \otimes S^{2} T^{*} \stackrel{\sigma_{1}(\beta) \oplus \gamma}{\longrightarrow} T^{*} \otimes T^{*} \oplus \mathbb{R} \longrightarrow 0
$$

is exact. Thus there is some obstruction to extending 3-jet solutions of the almost Kähler, Einstein equations. We wish to find out in more detail what this obstruction is.

The original technique for finding this obstruction in an explicit form used a good deal of spinors and representation theory. The interested reader could consult [5]. We shall take a different approach in this paper and identify the obstruction 
by more usual tensor methods. In fact we will obtain the relevant formulae in the case of manifolds with $J$-invariant Ricci tensor as well as just Einstein manifolds.

The above theory does not lead in a particularly simple way to finding the obstruction in an explicit form. It merely tells us that there is such an obstruction and that we will have to examine the 4-jets of the metric to find this obstruction - even though the obstruction will eventually take the form of a condition on the 3 -jets.

The calculations which follow do not rely on the above general theory - however, the authors would not have had any motivation to examine the 4-jets in such detail unless it was known that there was an interesting obstruction to be found and unless there were at least some clues as to where to look.

As a final remark, one might ask if there are any other obstructions to finding almost Kähler, Einstein metrics. It turns out that there are. In principal one could persistently prolong all the equations until all the obstructions have been found and the Cartan-Kähler theorem could be applied. We have attempted to do this, but gave in once two further obstructions were found.

\section{The explicit obstruction}

3.1. The self-dual Weyl tensor of an almost Kähler 4-manifold. Let $(M, g)$ be an oriented four-dimensional Riemannian manifold. We denote by $\Lambda^{2}=\Lambda^{+}$ $\oplus \Lambda^{-}$the bundle of real 2-forms on $M$, where $\Lambda^{+}$, resp. $\Lambda^{-}$, is the bundle of self-dual, resp. anti-self-dual, 2 -forms, i.e., the eigen-sub-bundle with respect to the eigenvalue +1 , resp. -1 , of the Hodge operator $*$, acting as an involution on $\bigwedge^{2}$. We will freely identify vectors and covectors via the metric $g$ and, accordingly, a 2-form $\phi$ with the corresponding skew-symmetric endomorphism of the tangent bundle $T$ by putting $g(\phi(X), Y)=\phi(X, Y)$, for any vector fields $X, Y$.

Considering the Riemannian curvature tensor $R$ as a symmetric endomorphism of $\bigwedge^{2}$ we have the following well known $\mathrm{SO}(4)$-splitting

$$
R=\frac{s}{12} \mathrm{Id}+\tilde{R i c}_{0}+W^{+}+W^{-},
$$

where $s$ is the scalar curvature, Ric $_{0}$ is the the Kulkarni-Nomizu extension of the traceless Ricci tensor $\mathrm{Ric}_{0}$ to an endomorphism of $\bigwedge^{2}$ anti-commuting with $*$, and $W^{ \pm}$are respectively the self-dual and anti-self-dual parts of the Weyl tensor $W$.

The self-dual Weyl tensor $W^{+}$of $(M, g)$ is viewed as a section of the bundle $S_{0}^{2}\left(\bigwedge^{+}\right)$of symmetric, traceless endomorphisms of $\bigwedge^{+}$(also considered as a subbundle of the tensor product $\left.\Lambda^{+} \otimes \Lambda^{+}\right)$.

Let $(M, g, J)$ be an almost Hermitian 4-manifold, i.e., an oriented Riemannian 4manifold $(M, g)$ endowed with an almost complex structure $J$ which is $g$-orthogonal $(g(J X, J Y)=g(X, Y))$ and positive (the orientation induced by $J$ coincides with the chosen orientation of $M)$. We denote by $\Omega$ the corresponding fundamental form defined by the equality $\Omega(X, Y)=g(J X, Y)$.

The action of $J$ extends to the cotangent bundle $T^{*}$ by putting $(J \alpha)(X)=$ $-\alpha(J X)$, so as to be compatible with the Riemannian duality between $T$ and $T^{*}$. This action defines an involution on $\bigwedge^{2}$, say $\imath_{J}$, acting by $\imath_{J}(\phi)(X, Y)=\phi(J X, J Y)$, which in turn gives rise to the following orthogonal splitting of $\Lambda^{+}$:

$$
\bigwedge^{+}=\mathbb{R} \Omega \oplus \llbracket \bigwedge^{0,2} \rrbracket
$$


where $\llbracket \bigwedge^{0,2} \rrbracket$ denotes the bundle of $J$-anti-invariant real 2-forms, equivalently, the 2 -forms $\phi$ satisfying $\imath_{J}(\phi)=-\phi$. Note that $\llbracket \wedge^{0,2} \rrbracket$ is the real underlying bundle of the anti-canonical bundle $\bigwedge^{0,2}$ of $(M, J)$; the induced complex structure $J$ on $\llbracket \wedge^{0,2} \rrbracket$ is then given by $(J \phi)(X, Y)=-\phi(J X, Y)$.

Consequently, the vector bundle $\mathcal{W}^{+}=S_{0}^{2}\left(\bigwedge^{+}\right)$of the symmetric traceless endomorphisms of $\bigwedge^{+}$decomposes into the sum of three sub-bundles, $\mathcal{W}_{1}^{+}, \mathcal{W}_{2}^{+}, \mathcal{W}_{3}^{+}$, defined as follows; see [23]:

- $\mathcal{W}_{1}^{+}=\mathbb{R}$ is the sub-bundle of elements preserving the decomposition (2) and acting by homothety on the two factors; hence is the trivial line bundle generated by the element $\frac{1}{8} \Omega \otimes \Omega-\frac{1}{12} \mathrm{Id}^{+}$.

- $\mathcal{W}_{2}^{+}=\llbracket \wedge^{0,2} \rrbracket$ is the sub-bundle of elements which exchange the two factors in (2); the real isomorphism with $\llbracket \wedge^{2,0} \rrbracket$ is seen by identifying each element $\phi$ of $\llbracket \bigwedge^{0,2} \rrbracket$ with the element $\frac{1}{2}(\Omega \otimes \phi+\phi \otimes \Omega)$ of $\mathcal{W}_{2}^{+}$.

- $\mathcal{W}_{3}^{+}=S_{0}^{2}\left(\llbracket \bigwedge^{0,2} \rrbracket\right)$ is the sub-bundle of elements preserving the splitting (2) and acting trivially on the first factor, $\mathbb{R} \Omega$.

Denote by $W_{i}^{+}, i=1,2,3$, the corresponding components of the positive Weyl tensor $W^{+}$; we then have

$$
W_{1}^{+}=\frac{\kappa}{8} \Omega \otimes \Omega-\frac{\kappa}{12} \mathrm{Id}^{+},
$$

where the smooth function $\kappa$ is the so-called conformal scalar curvature of $(g, J)$;

$$
W_{2}^{+}=-\frac{1}{4}(\Psi \otimes \Omega+\Omega \otimes \Psi)
$$

for a section $\Psi$ of $\llbracket \bigwedge^{0,2} \rrbracket$; for any (local) section $\phi$ of $\llbracket \bigwedge^{0,2} \rrbracket$ of square-norm 2 the component in $\mathcal{W}_{3}^{+}$is given by

$$
W_{3}^{+}=\frac{\lambda}{2}[\phi \otimes \phi-J \phi \otimes J \phi]+\frac{\mu}{2}[\phi \otimes J \phi+J \phi \otimes \phi],
$$

where $\lambda$ and $\mu$ are (locally defined) smooth functions.

For any almost Kähler structure $(g, J)$ the covariant derivative $\nabla \Omega$ of the Kähler form $\Omega$ is identified with the Nijenhuis tensor, the obstruction for integrability of the almost complex structure $J$. Furthermore, $\nabla \Omega$ can be viewed as a real section of the vector bundle $\bigwedge^{1,0} \otimes \bigwedge^{2,0}$, which allows us to write $\nabla \Omega$ (with respect to any section $\phi$ of $\left.\llbracket \bigwedge^{0,2} \rrbracket\right)$ in the following form:

$$
\nabla \Omega=a \otimes \phi-J a \otimes J \phi,
$$

where the 1-form $a$ satisfies $|\nabla \Omega|^{2}=4|a|^{2}$ provided that $\phi$ is of square-norm 2 . Consequently, the covariant derivatives of $\phi$ and $J \phi$ are given by

$$
\nabla \phi=-a \otimes \Omega+b \otimes J \phi ; \nabla J \phi=J a \otimes \Omega-b \otimes \phi,
$$

for some 1-form $b$.

Observe that into the formulas (5) and (6) we have a $S^{1}$-freedom for the choice of $\phi$. We shall refer to this as a gauge dependence.

From now on $\phi$ will be assumed to satisfy $\langle J \phi, \Psi\rangle=0$ at any point of $M$, or equivalently

$$
\Psi=\frac{|\Psi|}{\sqrt{2}} \phi,
$$


where $\Psi$ is the section of $\llbracket \Lambda^{0,2} \rrbracket$ determined by (4). Here, and henceforth, $\langle\cdot, \cdot \cdot\rangle$ denotes the extension of the metric $g$ to $\bigwedge^{2}$, such that the norm of a 2-form is the half of its usual tensor norm. We also put

$$
\Phi=W_{3}^{+}(\phi)=\lambda \phi+\mu J \phi
$$

so as $\Phi \equiv 0$ iff $W_{3}^{+} \equiv 0$ and $\Psi \equiv 0$ iff $W_{2}^{+} \equiv 0$ (see (5) and (4), respectively). We remark that most of our calculation will be made on the open dense subset $\mathcal{U} \subset M$ of the points $x$ where either $\Psi_{x} \neq 0$, or $\Psi \equiv 0$ in the neighbourhood of $x$. However, a standard continuity argument shows that the gauge independent global formulas obtained below will hold everywhere on $M$.

The self-dual Weyl tensor is completely determined by the scalar curvature and the second covariant derivative of the fundamental form $\Omega$ (equivalently, by the first covariant derivative of the Nijenhuis tensor). Indeed, once the gauge $\phi$ is fixed as above, it is sufficient to determine the smooth functions $\kappa,|\Psi|, \lambda$ and $\mu$ in terms of the 1-forms $a$ and $b$, and the 2-form $\phi$. For that we first make use of the Weitzenböck formula for self-dual 2-forms; cf. e.g. [8]:

$$
\Delta \psi=\nabla^{*} \nabla \psi+\frac{s}{3} \psi-2 W^{+}(\psi) .
$$

The self-dual 2 -form $\Omega$ is closed, and therefore harmonic; applying 10 to $\Omega$ we then get

$$
|\nabla \Omega|^{2}+\frac{2}{3} s-2\left\langle W^{+}(\Omega), \Omega\right\rangle=0
$$

and by (3)-(5) we conclude

$$
\kappa-s=6|a|^{2}=\frac{3}{2}|\nabla \Omega|^{2} .
$$

Formula (11) shows that the smooth function $\kappa-s$ is everywhere non-negative on $M$; it vanishes exactly at the points where the Nijenhuis tensor is zero.

In order to express $W_{2}^{+}$and $W_{3}^{+}$we make use of the Ricci identity:

$$
\left(\nabla_{X, Y}^{2}-\nabla_{Y, X}^{2}\right)(\Omega)(\cdot, \cdot)=-R_{X, Y}(J \cdot, \cdot)-R_{X, Y}(\cdot, J \cdot) .
$$

From (6) we get

$$
\left.\nabla^{2}\right|_{\wedge^{2}} \Omega=(d a-J a \wedge b) \otimes \phi-(d(J a)+a \wedge b) \otimes J \phi,
$$

so, 12) can be rewritten as

$$
d a-J a \wedge b=-R(J \phi) ; d(J a)+a \wedge b=-R(\phi) .
$$

Finally, by (11), (3)-(5), and (11) the equalities in (13) reduce to

$$
\begin{aligned}
\lambda & =-\frac{1}{2}\left(|a|^{2}-\langle d a, J \phi\rangle+\phi(a, b)\right) ; \\
\mu & =-\frac{1}{2}(\langle d a, \phi\rangle+J \phi(a, b)) ; \\
|\Psi| & =\sqrt{2}(\langle d(J a), \Omega\rangle+\Omega(a, b)) \\
& =-\sqrt{2}(\delta a-\Omega(a, b)) .
\end{aligned}
$$

We shall further analyse the above equalities involving the differential Bianchi identity. 
3.2. The differential Bianchi identity for almost Kähler 4-manifolds with $J$-invariant Ricci tensor. The co-differential $\delta W^{+}$of the self-dual Weyl tensor of $(M, g)$ is a section of the rank 8 vector bundle

$$
\mathcal{V}=\operatorname{Ker}\left(\operatorname{tr}: T^{*} \otimes \bigwedge^{+} \longrightarrow T^{*}\right),
$$

where $\operatorname{tr}$ is defined by $\operatorname{tr}(\alpha \otimes \phi)=\phi(\alpha)$ on decomposed elements. The vector bundle $\mathcal{V}$ splits as $\mathcal{V}=\mathcal{V}^{+} \oplus \mathcal{V}^{-}$(see [1]) where

(a) $\mathcal{V}^{+}$is identified with the cotangent bundle $T^{*}$ by

$$
\begin{gathered}
T^{*} \ni \alpha \mapsto A=J \alpha \otimes \Omega-\frac{1}{2} \sum_{i=1}^{4} e_{i} \otimes\left(\alpha \wedge e_{i}-J \alpha \wedge J e_{i}\right), \\
\mathcal{V}^{+} \ni A \mapsto \alpha=-\frac{1}{2} J\langle A, \Omega\rangle,
\end{gathered}
$$

where $\langle A, \Omega\rangle$ denotes the 1 -form defined by $X \mapsto\left\langle A_{X}, \Omega\right\rangle$.

(b) $\mathcal{V}^{-}$is identified, as a real vector bundle, to the vector bundle $\bigwedge^{0,1} \otimes \bigwedge^{0,2}$; if $\phi$ is a non-vanishing local section of $\llbracket \bigwedge^{0,2} \rrbracket$ satisfying $|\phi|^{2}=2$ at any point, then $\mathcal{V}^{-}$can be again identified with $T^{*}$ by

$$
\begin{aligned}
& \beta \in T^{*} \mapsto B=J \beta \otimes \phi+\beta \otimes J \phi, \\
& B \in \mathcal{V}^{-} \mapsto \beta=-\frac{1}{2} J\langle B, \phi\rangle .
\end{aligned}
$$

We denote by $\left(\delta W^{+}\right)^{+}$, resp. $\left(\delta W^{+}\right)^{-}$, the component of $\delta W^{+}$on $\mathcal{V}^{+}$, resp. on $\mathcal{V}^{-}$. Let $\alpha$ and $\beta$ be the corresponding 1 -forms, where $\beta$ is determined with respect to a local section $\phi$ of $\llbracket \wedge^{0,2} \rrbracket$ satisfying (8). By (17), (18), and (3)-(5) one directly calculates

$$
\begin{aligned}
\alpha=-\frac{1}{2} J\left\langle\delta W^{+}, \Omega\right\rangle=-\frac{d \kappa}{12}+\frac{1}{4} J \delta \Psi-\frac{|\Psi|}{4 \sqrt{2}} a-J \Phi(a) ; \\
\beta=\frac{1}{2}\left(-J\left\langle\delta W^{+}, \phi\right\rangle+\frac{1}{2} \phi\left\langle\delta W^{+}, \Omega\right\rangle\right) \\
=-\frac{\kappa}{8} a+\frac{1}{4 \sqrt{2}} d|\Psi|-\frac{1}{8} \phi(\delta \Psi)-\frac{1}{8} J \Psi(a) \\
-\frac{1}{2}(\Phi(b)+J \delta \Phi+\lambda a-\mu J a),
\end{aligned}
$$

where, we recall, the section $\Phi$, resp. $\Psi$, of $\llbracket \wedge^{0,2} \rrbracket$ is determined by (44) and (8), resp. by (5) and (9). Note that for any section $\psi$ of $\llbracket \wedge^{0,2} \rrbracket$ we have (see (6) $)$

$$
\delta(J \psi)=J \delta \psi+\langle\psi, \phi\rangle a-\langle\psi, J \phi\rangle J a .
$$

Moreover, by (6) and (7) we also get

$$
\delta \phi=J a-J \phi(b) ; \delta(J \phi)=a+\phi(b) .
$$

Taking into account (21), (8) and (22) we simplify (19) and (20) to the following:

Lemma 1. For any almost Kähler 4-manifold the 1-forms $\alpha$ and $\beta$ are given by

$$
\alpha=-\frac{d \kappa}{12}-\frac{3|\Psi|}{4 \sqrt{2}} a+\frac{1}{4} \delta(J \Psi)-J \Phi(a) ;
$$




$$
\beta=-\frac{\kappa}{8} a+\frac{1}{8 \sqrt{2}}(d|\Psi|+|\Psi| J b)-\frac{1}{2}(\Phi(b)+\delta(J \Phi)-\lambda a+\mu J a) .
$$

Recall that the Cotton-York tensor $C$ of $(M, g)$ is defined by

$$
C_{X, Y, Z}=\frac{1}{2}\left[\nabla_{Z}\left(\frac{s}{12} g+\operatorname{Ric}_{0}\right)(Y, X)-\nabla_{Y}\left(\frac{s}{12} g+\operatorname{Ric}_{0}\right)(Z, X)\right],
$$

for any vector fields $X, Y, Z$. Considering $C$ as a 2 -form with values in $T^{*}$, the second Bianchi identity reads as $\delta W=C$. In particular, we have the "half" Bianchi identity

$$
\delta W^{+}=C^{+},
$$

where $C^{+}$denotes the self-dual part of $C_{X}, X \in T$.

If the Ricci tensor is $J$-invariant, we make use of (23) to give an equivalent expression for the 1-forms $\alpha$ and $\beta$ in terms of the Ricci tensor and the 1-form $a$ defined by (6). According to (17)

$$
\begin{gathered}
\alpha(X)=-\frac{1}{2} J\left\langle C^{+}, \Omega\right\rangle=-\frac{1}{4} \sum_{i=1}^{4} \nabla_{e_{i}}\left(\frac{s}{12} g+\operatorname{Ric}_{0}\right)\left(J e_{i}, J X\right) \\
=-\frac{1}{4}\left[\frac{d s}{12}(X)-\left(\delta \operatorname{Ric}_{0}\right)(X)+\sum_{i=1}^{4} \operatorname{Ric}_{0}\left(e_{i}, J\left(\nabla_{e_{i}} J\right)(X)\right)-\operatorname{Ric}_{0}(\theta, X)\right] \\
=-\frac{1}{4}\left[\frac{d s}{3}(X)+\sum_{i=1}^{4} \operatorname{Ric}_{0}\left(e_{i}, J\left(\nabla_{e_{i}} J\right)(X)\right)\right] .
\end{gathered}
$$

Now using ([6) and the fact that the Ricci tensor is $J$-invariant, we get

$$
\sum_{i=1}^{4} \operatorname{Ric}_{0}\left(e_{i}, J\left(\nabla_{e_{i}} J\right)(X)\right)=0
$$

and we thus obtain

$$
\alpha=-\frac{d s}{12} \text {. }
$$

Regarding the component of $C^{+}$in $\mathcal{V}^{-}$, we have from (18)

$$
\beta=\frac{1}{2}\left(-J\left\langle C^{+}, \phi\right\rangle+\frac{1}{2} \phi\left\langle C^{+}, \Omega\right\rangle\right) .
$$

To compute $J\left\langle C^{+}, \phi\right\rangle$ we shall proceed in the same way as computing $J\left\langle C^{+}, \Omega\right\rangle$; instead of $J$ we consider the almost complex structure $I$, whose Kähler form is $\phi$. The traceless Ricci tensor $\mathrm{Ric}_{0}$ is now $I$-anti-invariant; involving (6), (7) and the expression for $\alpha$ that we have already obtained, we eventually get

$$
\beta=-\frac{1}{2} \operatorname{Ric}_{0}(a) .
$$

Comparing with Lemma 1 we obtain the following:

Lemma 2. (Differential Bianchi identity) Let $(M, g, J)$ be an almost Kähler 4manifold with $J$-invariant Ricci tensor. Then the 1 -forms $\alpha$ and $\beta$ are equivalently given by

$$
\alpha=-\frac{d s}{12}, \beta=-\frac{1}{2} \operatorname{Ric}_{0}(a) .
$$


In particular, the following relations hold:

$$
\begin{gathered}
d(\kappa-s)=-\frac{9 \sqrt{2}}{2}|\Psi| a+3 \delta(J \Psi)-12 J \Phi(a) ; \\
\operatorname{Ric}_{0}(a)=\frac{\kappa}{4} a-\frac{1}{4 \sqrt{2}}(d|\Psi|+|\Psi| J b)+(\Phi(b)+\delta(J \Phi)-\lambda a+\mu J a) .
\end{gathered}
$$

3.3. The co-differential of $\alpha$. Starting from Lemma 1, we may easily calculate the co-differential of the 1 -form $\alpha$ using the information we have from (14), (15) and (16). Specifically, we obtain

Lemma 3. For every almost Kähler 4-dimensional manifold the co-differential of $\alpha$ is given by either of the following two formulas:

$$
\begin{aligned}
\delta \alpha= & -\frac{\Delta \kappa}{12}-\frac{\kappa}{24}(\kappa-s)+\frac{3}{2}\left|W_{2}^{+}\right|^{2}-\left|W_{3}^{+}\right|^{2} \\
& +\frac{1}{2}\langle\nabla \Psi, \nabla \Omega\rangle-2 \beta(a) ; \\
\delta \alpha= & -\frac{\Delta \kappa}{12}+\frac{\kappa}{8}(\kappa-s)+\frac{3}{2}\left|W_{2}^{+}\right|^{2}-\left|W_{3}^{+}\right|^{2} \\
& -4\left(\Phi(a, b)-(\delta J \Phi)(a)+\lambda|a|^{2}\right)+6 \beta(a) .
\end{aligned}
$$

Proof. From (16) we get

$$
\delta(|\Psi| a)=|\Psi| \delta a-d|\Psi|(a)=-\frac{1}{\sqrt{2}}|\Psi|^{2}+(|\Psi| \Omega(a, b)-d|\Psi|(a)) .
$$

By (7) we further obtain

$$
d|\Psi|(a)-|\Psi| \Omega(a, b)=\frac{1}{\sqrt{2}}\langle\nabla \Psi, \nabla \Omega\rangle,
$$

where $\langle\cdot, \cdot\rangle$ also denotes the inner product on $T^{*} \otimes \Lambda^{2}$. These last two relations imply

$$
\delta(|\Psi| a)=-\frac{1}{\sqrt{2}}|\Psi|^{2}-\frac{1}{\sqrt{2}}\langle\nabla \Psi, \nabla \Omega\rangle .
$$

Clearly,

$$
\delta(J \Phi(a))=\langle d a, J \Phi\rangle-(\delta J \Phi)(a) .
$$

We make use of (14) and (15) to compute the first term of the right-hand side of the equality above, and (9), (21) and (22) for computing the second term. The final result is:

$$
\delta(J \Phi(a))=2\left(\lambda^{2}+\mu^{2}\right)+\left(\Phi(a, b)-(\delta J \Phi)(a)+\lambda|a|^{2}\right) .
$$

The norms of $W_{2}^{+}$and $W_{3}^{+}$(viewed as endomorphisms of $\bigwedge^{2}$ ) are easily calculated by (4) and (5), respectively:

$$
\left|W_{2}^{+}\right|^{2}=\frac{1}{4}|\Psi|^{2} ;\left|W_{3}^{+}\right|^{2}=2\left(\lambda^{2}+\mu^{2}\right) .
$$

It now follows from Lemma 1 and the equalities (26), (27) and (28) that

$$
\begin{aligned}
\delta \alpha= & -\frac{\Delta \kappa}{12}+\frac{3}{2}\left|W_{2}^{+}\right|^{2}-\left|W_{3}^{+}\right|^{2}+\frac{3}{8}\langle\nabla \Psi, \nabla \Omega\rangle \\
& -\left(\Phi(a, b)-(\delta J \Phi)(a)+\lambda|a|^{2}\right) .
\end{aligned}
$$


Finally, from the expression for $\beta$ claimed in Lemma 1 and by using (11), one can rewrite (29) in one of the forms (24) and (25).

Specifying Lemma 3 to the case of Einstein, almost Kähler 4-manifolds we obtain exactly the obstruction introduced in Section 2:

Proposition 1. For any almost Kähler 4-manifold with J-invariant Ricci tensor, the following relation holds:

$$
\begin{aligned}
\Delta(\kappa-s)= & -\frac{\kappa}{2}(\kappa-s)+18\left|W_{2}^{+}\right|^{2}-12\left|W_{3}^{+}\right|^{2} \\
& +6\langle\nabla \Psi, \nabla \Omega\rangle+12 \operatorname{Ric}_{0}(a, a) .
\end{aligned}
$$

In the case of an Einstein manifold, this condition on the 3-jet of an almost Kähler 4-manifold is precisely the obstruction referred to earlier. Moreover, for any almost Kähler 4-manifold with J-invariant Ricci tensor and Hermitian Weyl tensor we further have

$$
\Delta(\kappa-s)=\frac{3 \kappa}{2}(\kappa-s)+18\left|W_{2}^{+}\right|^{2}-36 \operatorname{Ric}_{0}(a, a) .
$$

Proof. Equality (30) follows by Lemma 3, 24) and Lemma 2 while the relation (31) follows from Lemma 3, (25), specified to the case when $\Phi \equiv 0$ (see (9)), and Lemma 2.

Remark 1. The relation (30) is already apparent in the literature; see e.g. [12. Lemma 2]. In the case of a compact Einstein almost Kähler 4-manifold, after integrating (30) one obtains exactly the integral formula of Sekigawa [22]

$$
\int_{M}\left(\kappa(\kappa-s)+12\left|W_{2}^{+}\right|^{2}+24\left|W_{3}^{+}\right|^{2}\right) d V_{g}=0 .
$$

Since the function $\kappa-s$ is everywhere non-negative (see (11)), it follows from (32) that in the case of non-negative scalar curvature we have $\kappa=s, i . e .,(g, J)$ is Kähler 22 .

Remark 2. A companion result of Proposition 1. (30) can be obtained as an application of the Weitzenböck formula for 2 -forms with values in $T^{*}$ (cf. e.g. [9]); specifying for a section $V$ of the sub-bundle $\mathcal{V}^{-}$we obtain

$$
2\left\langle d^{\nabla} \delta^{\nabla} V, V\right\rangle=\frac{1}{2} \Delta|V|^{2}+|\nabla V|^{2}+\frac{(5 s+4 \kappa)}{12}|V|^{2}+\left\langle\operatorname{Ric}_{0}, S^{V}\right\rangle,
$$

where $S_{X, Y}^{V}=\left\langle V_{X}, V_{Y}\right\rangle$. For an almost Kähler 4-manifold we have a distinguished section of $\mathcal{V}^{-}$, the the Nijenhuis tensor $\nabla \Omega$. If, moreover, the Ricci tensor is $J$ invariant, then the Weitzenböck formula for $\nabla \Omega$ reads as (compare with [12] Lemma $1])$ :

$$
\begin{aligned}
\Delta(\kappa-s)= & -\frac{1}{4}(\kappa+5 s)(\kappa-s)-6\left|W_{3}^{+}\right|^{2}-6\left|W_{2}^{+}\right|^{2}-3\langle\nabla \Psi, \nabla \Omega\rangle \\
& -12 \operatorname{Ric}_{0}(a, a)-3\left|\left(\nabla^{2} \Omega\right)_{0}^{s y m}\right|^{2},
\end{aligned}
$$

where $\left(\nabla^{2} \Omega\right)_{0}^{\text {sym }}$ denotes the symmetric traceless part of $\nabla^{2} \Omega$, considered as a section of $T^{*} \otimes T^{*} \otimes \bigwedge^{2}$. The "difference" between (30) and 34 gives another "curvature obstruction" for existence of compact, strictly almost Kähler 4-manifolds with $J$-invariant Ricci tensor. In the case when $M$ is compact and the scalar curvature is non-negative it follows from (30) and (34) that the almost Kähler structure is necessarily Kähler; cf. [12. 


\section{Proof OF RESUlts}

4.1. Proof of Theorem [1. As $\mathrm{Ric}_{0}$ is a $J$-invariant, traceless and symmetric tensor its eigenvalues at any point are equal to

$$
\left(\frac{\left|\mathrm{Ric}_{0}\right|}{2}, \frac{\left|\mathrm{Ric}_{0}\right|}{2},-\frac{\left|\mathrm{Ric}_{0}\right|}{2},-\frac{\left|\mathrm{Ric}_{0}\right|}{2}\right),
$$

where $\left|\mathrm{Ric}_{0}\right|$ denotes the norm of $\mathrm{Ric}_{0}$; by using (11) we obtain the following pointwise inequality:

$$
\operatorname{Ric}_{0}(a, a) \leq \frac{\left|\operatorname{Ric}_{0}\right|}{2}|a|^{2}=\frac{\left|\operatorname{Ric}_{0}\right|}{12}(\kappa-s)
$$

Since the function $\left|\mathrm{Ric}_{0}\right|$ is continuous on $M$, there is a positive constant $A>0$, such that $\left|\operatorname{Ric}_{0}\right| \leq A$ everywhere on $M$. Then Corollary 1, (31) implies

$$
\Delta(\kappa-s)+\left(3 A-\frac{3 \kappa}{2}\right)(\kappa-s) \geq 0
$$

Now, from the maximal principle (see e.g. [6, Prop. 3.75]) we infer that either $\kappa-s$ identically vanishes (i.e. $(g, J)$ is Kähler), or else $\kappa-s$ is everywhere positive on $M$. In the latter case it follows from [3] that

$$
2 c_{1}^{2}(M)+e(M)=0,
$$

where $c_{1}^{2}(M)$ denotes the first Chern number of $(M, J)$. Now our claim follows by the well known formula of $\mathrm{Wu}: c_{1}^{2}(M)=2 e(M)+3 \sigma(M)$.

4.2. Proof of Corollary 1. Assume that $(M, g, J)$ is Einstein, non-Kähler with Hermitian Weyl tensor. By Theorem 1 we get

$$
5 e(M)+6 \sigma(M)=0 .
$$

On the other hand, for every compact oriented, Einstein 4-manifold the Chern-Weil formulae read as

$$
\begin{gathered}
e(M)=\frac{1}{8 \pi^{2}} \int_{M}\left(\left|W^{+}\right|^{2}+\left|W^{-}\right|^{2}+\frac{s^{2}}{24}\right) d V_{g}, \\
\sigma(M)=\frac{1}{12 \pi^{2}} \int_{M}\left(\left|W^{+}\right|^{2}-\left|W^{-}\right|^{2}\right) d V_{g} .
\end{gathered}
$$

Substituting into (34) we obtain $s=0, W^{+}=0, W^{-}=0$, i.e., $g$ is flat. But then $(g, J)$ must be Kähler-flat because we have $\kappa=s=0$ (see (11)), a contradiction.

\section{Almost Kähler 4-Manifolds and Lagrangian sectional CURVAture}

For some time the question of existence of non-Kähler, almost Kähler 4-manifolds of constant sectional curvature has been open. Now the answer is known to be negative, as a consequence of the results in [4, 19.

In this section we use Theorem [1 to study a related problem - existence of almost Kähler 4-manifolds $(M, g, J)$ whose sectional curvature at any point is constant on the Lagrangian (with respect to the symplectic form $\Omega$ ) 2-planes. One may consider this condition as a symplectic counterpart of the notion of constant holomorphic sectional curvature in the Kähler geometry. A simple local calculation shows that for any such manifold the Ricci tensor is $J$-invariant, and the Weyl tensor is Hermitian and self-dual (see [13], or [2] Lemma 5]). For Kähler surfaces these conditions simply reduce to the vanishing of the anti-self-dual Weyl tensor; 
the compact ones with this property are completely described in [10] (see also [9], [11], [18]). Given a compact symplectic 4-manifold $(M, \Omega)$ it is plausible to conjecture [2] that a compatible almost Kähler structure $(g, J)$ of pointwise constant sectional curvature on the Lagrangian 2-planes is, in fact, a self-dual Kähler structure. The conjecture has been confirmed in [2] under the additional assumption that the scalar curvature is constant.

Theorem 1 in turn gives strong evidence that the conjecture seems to be true. If there exists a compact strictly almost Kähler 4-manifold $(M, g, J)$ whose sectional curvature is pointwise constant on the Lagrangian 2-planes, then the Chern-Weil formula for the signature and Theorem 1 show that the Euler characteristic of $M$ must be strictly negative while the Betti number $b^{+}(M)$ must be greater than 1; to the authors' knowledge there are no examples of compact 4-dimensional symplectic manifolds satisfying the these topological assumptions. At least in the case that $M$ admits a complex structure compatible with the orientation induced by $J$ (but which does not need to be compatible neither with metric nor with the symplectic form) a simple argument relying on the classification of compact complex surfaces leads to the following:

Corollary 2. Let $(M, g, J)$ be compact 4-dimensional almost Kähler manifold of pointwise constant sectional curvature on the Lagrangian 2-planes. Suppose that the underlying oriented manifold $M$ (considered with the canonical orientation induced by $J$ ) is orientedly homotopic to a compact complex surface. Then $J$ is integrable, i.e., $(g, J)$ is a self-dual Kähler structure on $M$.

Proof. Suppose $(M, g, J)$ is an almost Kähler 4-manifold of pointwise constant Lagrangian sectional curvature. A simple local calculation shows that the metric $g$ is self-dual with $J$-invariant Ricci tensor, and with Hermitian Weyl tensor; cf. [13] (see also [2, Lemma 5]). Assume that $(g, J)$ is not Kähler. Since $g$ is self-dual, the Chern-Weil formula

$$
\sigma(M)=\frac{1}{12 \pi^{2}} \int_{M}\left(\left|W^{+}\right|^{2}-\left|W^{-}\right|^{2}\right) d V_{g}=\frac{1}{12 \pi^{2}} \int_{M}\left|W^{+}\right|^{2} d V_{g}
$$

shows that $\sigma(M) \geq 0$. If $\sigma(M)=0$, we have $W^{+}=0$ and $J$ is then integrable by [2. Cor. 1], a contradiction. We thus have $\sigma(M)>0$, and, by Theorem 1, we conclude that $e(M)<0$.

Let $S$ be a complex surface which is orientedly homotopic to $M$. It is a well known result of Grauert that every compact complex surface can be obtain from a minimal one (called minimal model) by successive blowing ups. It is also well known that by blowing up a compact complex surface at a point its Euler characteristic increases by one while its signature decreases by one. Thus, if we denote by $S_{0}$ a minimal model of $S$, we have $e\left(S_{0}\right)<0$ and $\sigma\left(S_{0}\right)>0$. By the Kodaira classification of complex surfaces (cf. e.g. [7]) the condition $e\left(S_{0}\right)<0$ implies that $S_{0}$ is the projectivization of a holomorphic rank 2 vector bundle over a compact curve of genus at least 2 . But then the signature of $S_{0}$ is zero, a contradiction.

\section{ACKNOWLEDGEMENTS}

The first-named author thanks the Mathematical Institute of Oxford for its generous hospitality during the preparation of this work. He is also grateful to P. Gauduchon for his interest and suggestions, T. Draghici for his friendly assistance in 
carefully reading the manuscript and suggesting some valuable improvements, and D. Kotschick for some helpful discussions on the geography of compact 4-manifolds.

\section{REFERENCES}

1. V. Apostolov and P. Gauduchon, The Riemannian Goldberg-Sachs Theorem, Int. J. of Math. 8 (1997), 421-439. MR 98g:53080

2. V. Apostolov and T. Draghici, Almost Kähler 4-manifolds with J-invariant Ricci tensor and special Weyl tensor, Quart. J. Math. Oxford Ser.(2), to appear.

3. J. Armstrong, On four-dimensional almost Kähler manifolds, Quart. J. Math. Oxford Ser.(2) 48 (1997), 405-415. MR 98k:53054]

4. J. Armstrong, An Ansatz for Almost-Kähler, Einstein 4-manifolds, preprint, 1997.

5. J. Armstrong, Almost Kähler Geometry, Ph.D. Thesis, Oxford, 1998.

6. T. Aubin, Non-linear Analysis on Manifolds. Monge-Ampère Equations, Springer-Verlag, Grund. Math. Wiss., 252, 1982. MR 85j:58002

7. W. Barth, C. Peters, and A. Van de Ven, Compact complex surfaces, Springer-Verlag, 1984. MR 86c:32026

8. J.-P. Bourguignon, Formules de Weitzenböck en dimension 4, in Géométrie riemannienne en dimension 4, Séminaire Arthur Besse, 1978/79, eds. Bérard-Bergery, M. Berger, C. Houzel, Cedic/Fernand Nathan, Paris 1981. CMP 17:05

9. J.-P. Bourguignon, Les variétés de dimension 4 à signature non nule dont la courbure est harmonique sont d'Einstein, Invent. Math. 63 (1981), 263-286. MR 82g:5305

10. B.-Y. Chen, Some topological obstructions to Bochner-Kähler metrics and their applications, J. Diff. Geom. 13 (1978), 574-588. MR 81f:32037

11. A. Derdziński, Self-dual Kähler manifolds and Einstein manifolds of dimension four, Compositio Math. 49 (1983), 405-433. MR 84h:53060

12. T. Draghici, Almost Kähler 4-manifolds with J-invariant Ricci tensor, Houston J. of Math., Vol.25, no.1, (1999), 133-145. CMP 99:13

13. G. Ganchev, On Bochner curvature tensors in almost Hermitian manifolds, Pliska Studia Matematica Bulgarica 9 (1987), 33-43. MR 88e:53045

14. J. Gasqui, Sur la résolubilité locale des équations d'Einstein, Compositio Math. 47 (1982), 43-69. MR 84f:58115

15. J. Goldberg and R. Sachs, A theorem on Petrov types, Acta Phys. Polon. 22 Suppl. (1962), 13-23. MR 27:6599

16. S.I. Goldberg, Integrability of almost Kähler manifolds, Proc. Amer. Math. Soc. 21 (1969), 96-100. MR 38:6514

17. H. Goldschmidt, Integrability criteria for systems of non-linear partial differential equations, J. Diff. Geom. 1 (1967), 269-307. MR 37:1746

18. M. Itoh, Self-duality of Kähler surfaces, Compositio Math. 51 (1984), 265-273. MR 85m:53079

19. T. Oguro and K. Sekigawa Non-existence of almost Kähler structure on hyperbolic spaces of dimension $2 n(\geq 4)$, Math. Ann. 300 (1994), 317-329. MR 95h:53066

20. M. Przanowski and B. Broda, Locally Kähler gravitational instantons, Acta Phys. Polon. B 14 (1983), 637-661. MR 85g:83016b

21. I. Robinson and A. Schild, A generalization of a theorem by Goldberg and Sachs, J. Math. Phys. 4 (1963), 484. MR 26:7421

22. K. Sekigawa, On some 4-dimensional compact Einstein almost Kähler manifolds, Math. Ann. 271 (1985), 333-337. MR 86g:53054

23. F. Tricerri and L.Vanhecke Curvature tensors on almost Hermitian manifods, Trans. Amer. Math. Soc. 267 (1981), 365-398. MR 82j:53071

IHÉS - EPDi, Le Bois-Marie, 35, route de Chartres, F-91440 Bures-Sur-Yvette Cedex, FRANCE

E-mail address: apostolo@ihes.fr

10 Alan Bullock Close, Oxford OX4 1AU, United Kingdom

E-mail address: John.Armstrong@madge.com 